\title{
OPEN Dietary glutamic acid and aspartic acid as biomarkers for predicting diabetic retinopathy
}

\author{
So Young Park ${ }^{1,6}$, Jieun Kim ${ }^{2,6}$, Jung II Son ${ }^{3}$, Sang Youl Rhee ${ }^{4 凶}$, Do-Yeon Kim², Suk Chon ${ }^{4}$, \\ Hyunjung Lim $^{5 \bowtie}$ \& Jeong-Taek Woo ${ }^{4}$
}

The screening rate of diabetic retinopathy (DR) is low despite the importance of early diagnosis. We investigated the predictive value of dietary glutamic acid and aspartic acid for diagnosis of DR using the Korea National Diabetes Program cohort study. The 2067 patients with type 2 diabetes without DR were included. The baseline intakes of energy, glutamic acid and aspartic acid were assessed using a 3-day food records. The risk of DR incidence based on intake of glutamic acid and aspartic acid was analyzed. The DR group was older, and had higher HbA1c, longer DM duration, lower education level and income than non-DR group (all $p<0.05$ ). The intake of total energy, glutamic acid and aspartic acid were lower in DR group than non-DR group $(p=0.010, p=0.025$ and $p=0.042$, respectively). There was no difference in the risk of developing DR according to the intake of glutamic acid and ascorbic acid. But, aspartic acid intake had a negative correlation with PDR. Hence, the intake of glutamic acid and aspartic acid did not affect in DR incidence. However, lower aspartic acid intake affected the PDR incidence.

Diabetic retinopathy (DR) is a common microvascular complication associated with diabetes. The reported global prevalence of DR in type 2 diabetes mellitus (T2DM) patients is $30-40 \%{ }^{1}$. Because DR is more specific to hyperglycemia than other diabetic complications, it is referenced in the diagnosis of $\mathrm{DM}^{2}$. Chronic hyperglycemia induced retinal vascular endothelial dysfunction causes retinal ischemia and increased vascular permeability, resulting in vision-threatening $\mathrm{DR}^{3}$. Moreover, DR accounts for $4.8 \%$ of blindness ${ }^{4}$. And as diabetes prevalence increases worldwide, DR is considered a leading cause of visual loss ${ }^{5}$. Visual impairment due to DR can not only reduce patient quality of life but also increase medical expenses and result in substantial burden to the national healthcare system. While early detect and proper management of DR can prevent severe vision loss or blindness ${ }^{3}$, the screening rate for DR is significantly lower than that for other complications ${ }^{6}$ because the specific instrument such as fundus camera and skilled physician are required for screening ${ }^{7}$. Therefore, previous studies have sought to identify effective biomarkers easier screening for DR as well as predicting treatment response and prognosis. However, the biomarkers already used widely in clinical research and practice lack accurate prediction; thus, residual risk remains ${ }^{8}$.

Our recent metabolomics study in a well-organized cohort of geriatric diabetic patients showed that some important metabolites such as plasma glutamic acid, and aspartic acid, etc. were directly related to $\mathrm{DR}^{9}$. However, the study was cross-sectional in design; thus, it was difficult to identify causal relationships. Therefore, we sought to obtain more clear evidence using large-scale, multicenter, prospective cohort data. This study aimed to investigate the causal relationship between intakes of glutamic and aspartic acids and DR based on an analysis of large-scale, multicenter, prospective cohort data. We also classified non-proliferative diabetic retinopathy (NPDR) and proliferative diabetic retinopathy (PDR) according to DR severity and analyzed the relationship between DR severity and dietary glutamic acid and aspartic acid.

\footnotetext{
${ }^{1}$ Department of Endocrinology \& Metabolism, Kyung Hee University Hospital, Seoul, Republic of Korea. ${ }^{2}$ Research Institute of Medical Nutrition, Kyung Hee University, Seoul, Republic of Korea. ${ }^{3}$ Il San Gospel Hospital, Goyang-si, Gyeonggi-do, Republic of Korea. ${ }^{4}$ Department of Endocrinology and Metabolism, Kyung Hee University School of Medicine, 23 Kyungheedae-ro, Dongdaemun-gu, Seoul 02447, Republic of Korea. ${ }^{5}$ Department of Medical Nutrition, Graduate School of East-West Medical Science, Kyung Hee University, 1732 Deogyeong-daero, Giheung-gu, Yongin 17104, Republic of Korea. ${ }^{6}$ These authors contributed equally: So Young Park and Jieun Kim. ${ }^{\boxplus}$ email: rheesy@khu.ac.kr; hjlim@khu.ac.kr
} 


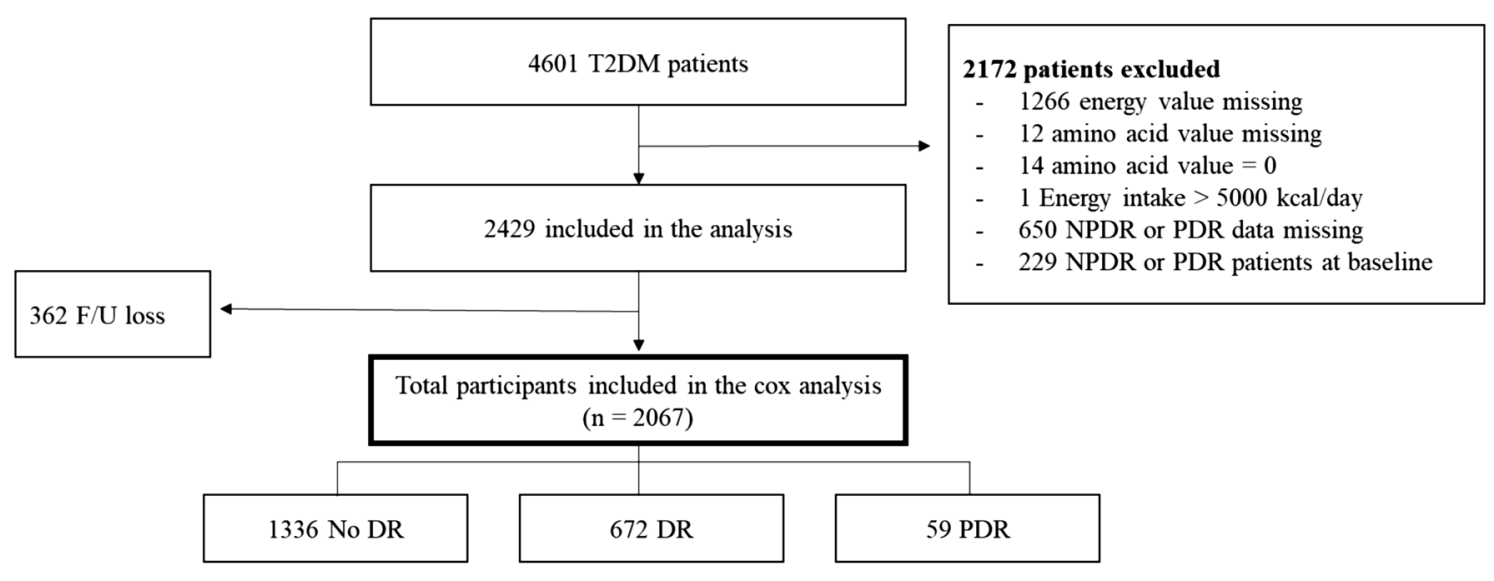

Figure 1. Study progression. $D M$ diabetes mellitus; $N P D R$ non-proliferative diabetic retinopathy; $P D R$ proliferative diabetic retinopathy; $D R$ diabetic retinopathy.

\section{Results}

Baseline subject characteristics. Of the 2429 subjects recruited to the study, 2067 completed the study, including 731 (35.4\%) diagnosed with DR; 672 (32.5\%) with NPDR, and 59 (2.9\%) with PDR (Fig. 1).

The baseline characteristics of the subjects by DR status are shown in Table 1 . The DR group was older $(p<0.0001)$, and had a higher glycated hemoglobin (HbA1c) $(p<0.0001)$, longer DM duration $(p<0.0001)$, lower education level $(p=0.011)$, and lower income $(p=0.001)$ than those in the non-DR group. Although all values were within the normal ranges, the alanine aminotransferase (ALT) was lower $(p=0.007)$, and blood urea nitrogen (BUN) were higher $(p=0.046)$ in the DR group than those in the non-DR group. The low-density lipoprotein (LDL) cholesterol level was higher and the high-density lipoprotein (HDL) cholesterol level was lower in the DR group ( $p=0.013$ and $p=0.025$, respectively). The DR group had a higher proportion of participants with a history of hypertension (HTN) and cerebrovascular disease ( $p=0.004$ and $p=0.002$, respectively). The total energy, glutamic acid, and aspartic acid intakes were lower in the DR group than those in the non-DR group $(p=0.010, p=0.025$, and $p=0.042$, respectively) (Fig. 2). Among subjects with DR, no difference in HbAlc was observed between the NPDR and PDR groups; however, the PDR group had a longer DM duration than that in the NPDR group. Renal dysfunctions and lower aspartic acid intake were observed in the PDR group compared to those in the NPDR group.

The effect of dietary glutamic and aspartic acid on DR incidence. The median follow-up period was 5 years. The incidence of DR was 210.4 cases/1000 person/year; NPDR, 193.4 cases/1000 person/year; and PDR, 17.0 cases/1000 person/year (Table 2).

The absolute intakes and proportions of total protein intake of glutamic acid and aspartic acid were divided into tertiles and the DR risk according to tertiles of absolute intake of glutamic acid and aspartic acid was analyzed (Table 3). The hazard ratio (HR) of glutamic acid for DR was 1.00 [95\% confidence interval (CI) 0.78-1.28] in the middle tertile, and 0.90 (95\% CI 0.66-1.22) in the highest tertile compared to the lowest tertile. The HR of aspartic acid for DR was 1.04 (95\% CI 0.81-1.34) in the middle tertile, and 0.90 (95\% CI 0.66-1.23) in the highest tertile compared to the lowest tertile. After adjusting for confounding factors, no difference in DR risk was observed according to tertiles of absolute intake ( $\mathrm{mg}$ ) of glutamic acid and aspartic acid. However, the absolute intake of aspartic acid was associated with PDR. The HR of aspartic acid for PDR was 0.12 (95\% CI 0.02-0.98) in the highest tertile compared to the lowest tertile. The results remained consistent after adjusting for confounding factors. Table 4 shows the DR risk according to tertiles of the proportions of total protein intake of glutamic and aspartic acids. These proportions of total protein intake also did not affect the risk of DR. However, the negative effect of aspartic acid on PDR was more pronounced. The HR of aspartic acid for PDR was 0.43 (95\% CI $0.19-0.94)$ in the middle tertile, and 0.43 (95\% CI 0.20-0.94) in the highest tertile compared to the lowest tertile. The results remained consistent after adjustment.

\section{Discussion}

In our study, the patients with incident DR were older and had a higher HbA1c level, longer DM duration, higher LDL cholesterol level, and lower HDL cholesterol level than those in patients without incident DR. Additionally, more patients in the DR group had a history of HTN and cerebrovascular diseases. Uncontrolled $\mathrm{DM}, \mathrm{HTN}$, dyslipidemia, and long diabetes duration are well-known risk factors for $\mathrm{DR}^{1,13}$. In the present study, more patients in the DR group had cerebrovascular disease compared to the non-DR group, but no difference in cardiovascular disease was observed. Previous studies reported increased risks of cardiovascular disease in patients with $\mathrm{DR}^{14,15}$. However, as cerebrovascular disease better reflects microangiopathy involving small vessel diseases than cardiovascular disease ${ }^{16}$, more patients with DR might have cerebrovascular disease. The lower educational and economic state in the DR group may be associated with low availability of medical services.

Our previous cross-sectional study demonstrated plasma glutamine, glutamic acid, and their ratio as predictors of DR ${ }^{9}$. High plasma glutamine level and glutamine/glutamic acid ratio and low plasma glutamic acid level 


\begin{tabular}{|c|c|c|c|c|c|c|}
\hline & \multirow[b]{2}{*}{ No $D R(n=1336)$} & \multicolumn{4}{|l|}{$\mathrm{DR}(\mathrm{n}=731)$} & \multirow[b]{2}{*}{$p$ value (no DR vs DR) } \\
\hline & & NPDR $(n=672)$ & PDR $(n=59)$ & $p$ value (NPDR vs PDR) & $\mathrm{DR}(\mathrm{n}=731)$ & \\
\hline Age (years) & $53.1 \pm 9.7$ & $55.5 \pm 9.6$ & $56.1 \pm 11.0$ & 0.678 & $55.6 \pm 9.7$ & $<0.0001$ \\
\hline Gender (n, \%) & & & & & & 0.245 \\
\hline Men & $781(58.5)$ & $375(55.8)$ & $33(55.9)$ & 0.985 & $408(55.8)$ & \\
\hline Women & $555(41.5)$ & $297(44.2)$ & $26(44.1)$ & & $323(44.2)$ & \\
\hline DM duration (years) & $5.5 \pm 5.5$ & $7.7 \pm 6.7$ & $11.9 \pm 8.6$ & 0.008 & $8.0 \pm 7.0$ & $<0.0001$ \\
\hline \multicolumn{7}{|l|}{ Education (n, \%) } \\
\hline Middle school $\leq$ & $433(34.3)$ & $257(39.1)$ & $23(41.1)$ & 0.329 & $280(39.2)$ & 0.011 \\
\hline High school & $451(35.7)$ & $239(36.3)$ & $24(42.9)$ & & $263(36.8)$ & \\
\hline College/University & $378(30.0)$ & $162(24.6)$ & $9(16.1)$ & & $171(24.0)$ & \\
\hline Income, won/month (n, \%) & & & & & & 0.001 \\
\hline$\leq 200 \times 10^{4}$ & $433(35.9)$ & $268(42.7)$ & $27(50.9)$ & 0.208 & $295(43.3)$ & \\
\hline$<201 \times 10^{4}$ and $\leq 400 \times 10^{4}$ & $412(34.2)$ & $212(33.8)$ & $19(35.9)$ & & $231(33.9)$ & \\
\hline$>401 \times 10^{4}$ & $360(29.9)$ & $148(23.4)$ & $7(13.2)$ & & $155(22.8)$ & \\
\hline Occupation (n, \%) & & & & 0.005 & & 0.423 \\
\hline Administration, office workers & $234(18.7)$ & $108(16.5)$ & $5(9.3)$ & & $113(16.0)$ & \\
\hline $\begin{array}{l}\text { Sales, service, agricultural, laborer, piscato- } \\
\text { rial }\end{array}$ & $276(22.1)$ & $133(20.3)$ & $15(27.8)$ & & $148(20.9)$ & \\
\hline Self-employed & $169(13.5)$ & $94(14.4)$ & $4(7.4)$ & & $98(13.8)$ & \\
\hline Homemaker & $379(30.3)$ & $106(16.2)$ & $18(33.3)$ & & $225(31.8)$ & \\
\hline Student, not employed, army & $192(15.4)$ & $213(32.6)$ & $12(22.2)$ & & $124(17.5)$ & \\
\hline Smoking $(\mathrm{n}, \%)$ & & & & 0.523 & & 0.055 \\
\hline Current & $302(22.7)$ & $131(19.5)$ & $9(15.3)$ & & $140(19.2)$ & \\
\hline Ex-smoker & $380(28.5)$ & $181(27.0)$ & $14(23.7)$ & & $195(26.7)$ & \\
\hline Never & $651(48.8)$ & $359(53.5)$ & $36(61.0)$ & & $395(54.1)$ & \\
\hline Alcohol (n, \%) & & & & 0.547 & & 0.057 \\
\hline Current & $702(52.7)$ & $320(47.8)$ & $26(44.1)$ & & $346(47.5)$ & \\
\hline Ex-drinker & $128(9.6)$ & $62(9.3)$ & $8(13.6)$ & & $70(9.6)$ & \\
\hline Never & $503(37.7)$ & $288(43.0)$ & $25(42.4)$ & & $313(42.9)$ & \\
\hline BMI $\left(\mathrm{kg} / \mathrm{m}^{2}\right)$ & $25.2 \pm 0.1$ & $25.0 \pm 0.1$ & $24.9 \pm 0.4$ & 0.648 & $25.0 \pm 0.1$ & 0.345 \\
\hline SBP (mmHg) & $124.8 \pm 0.4$ & $125.3 \pm 0.6$ & $128.5 \pm 2.0$ & 0.112 & $125.3 \pm 0.5$ & 0.454 \\
\hline DBP (mmHg) & $78.1 \pm 0.3$ & $77.6 \pm 0.4$ & $77.9 \pm 1.3$ & 0.810 & $77.8 \pm 0.4$ & 0.498 \\
\hline HbAlc (\%) & $7.6 \pm 0.0$ & $8.0 \pm 0.1$ & $8.2 \pm 0.2$ & 0.364 & $8.0 \pm 0.0$ & $<0.0001$ \\
\hline $\mathrm{TC}(\mathrm{mg} / \mathrm{dL})$ & $178.6 \pm 1.1$ & $181.6 \pm 1.6$ & $182.8 \pm 5.5$ & 0.833 & $182.1 \pm 1.5$ & 0.063 \\
\hline TG (mg/dL) & $159.3 \pm 3.4$ & $157.4 \pm 4.5$ & $137.2 \pm 15.8$ & 0.222 & $158.2 \pm 4.6$ & 0.837 \\
\hline HDL-C (mg/dL) & $48.3 \pm 0.4$ & $47.0 \pm 0.5$ & $47.1 \pm 1.8$ & 0.962 & $46.9 \pm 0.5$ & 0.025 \\
\hline LDL-C (mg/dL) & $99.8 \pm 1.0$ & $103.8 \pm 1.5$ & $106.3 \pm 5.3$ & 0.661 & $104.2 \pm 1.4$ & 0.013 \\
\hline BUN (mg/dL) & $14.6 \pm 0.1$ & $15.1 \pm 0.2$ & $15.5 \pm 0.7$ & 0.530 & $15.0 \pm 0.2$ & 0.046 \\
\hline Creatinine (mg/dL) & $0.8 \pm 0.0$ & $0.8 \pm 0.0$ & $0.9 \pm 0.0$ & 0.016 & $0.8 \pm 0.0$ & 0.321 \\
\hline $\mathrm{CrCl}(\mathrm{mL} / \mathrm{min})$ & $94.5 \pm 0.8$ & $94.9 \pm 1.2$ & $85.1 \pm 4.1$ & 0.020 & $96.6 \pm 1.0$ & 0.113 \\
\hline AST (IU/l) & $26.1 \pm 0.4$ & $25.0 \pm 0.5$ & $23.3 \pm 1.8$ & 0.374 & $25.0 \pm 0.5$ & 0.094 \\
\hline ALT (IU/l) & $30.2 \pm 0.6$ & $27.5 \pm 0.7$ & $25.9 \pm 2.5$ & 0.546 & $28.0 \pm 0.8$ & 0.034 \\
\hline \multicolumn{7}{|l|}{ Comorbidity (n, \%) } \\
\hline Hypertension & $618(46.3)$ & 360 (53.7) & $34(57.6)$ & 0.724 & 394 (53.0) & 0.004 \\
\hline Dyslipidemia & $591(44.2)$ & $289(43.1)$ & $25(42.4)$ & 0.974 & $314(43.0)$ & 0.808 \\
\hline Cardiovascular disease & $72(5.4)$ & $45(6.8)$ & $7(11.9)$ & 0.148 & $52(7.2)$ & 0.110 \\
\hline Cerebrovascular disease & $50(3.8)$ & $48(7.2)$ & $2(3.4)$ & 0.266 & $50(6.9)$ & 0.002 \\
\hline \multicolumn{7}{|l|}{ Dietary intake } \\
\hline Energy (kcal) & $1816.6 \pm 375.9$ & $1,774.2 \pm 377.5$ & $1,741.7 \pm 403.5$ & 0.529 & $1771.6 \pm 379.5$ & 0.010 \\
\hline Glutamic acid (mg) & $11,149.0 \pm 3594.5$ & $10,851.5 \pm 3488.5$ & $10,011.9 \pm 3127.2$ & 0.074 & $10,783.7 \pm 3466.3$ & 0.025 \\
\hline Glutamic acid (\% total protein) & $14.2 \pm 2.5$ & $14.2 \pm 2.5$ & $13.8 \pm 3.1$ & 0.241 & $14.1 \pm 2.5$ & 0.315 \\
\hline Aspartic acid (mg) & $6142.0 \pm 2072.7$ & $6009.9 \pm 2052.6$ & $5252.8 \pm 1669.9$ & 0.007 & $5948.8 \pm 2034.0$ & 0.042 \\
\hline Aspartic acid (\% total protein) & $7.8 \pm 1.6$ & $7.8 \pm 1.5$ & $7.2 \pm 1.7$ & 0.005 & $7.8 \pm 1.6$ & 0.395 \\
\hline
\end{tabular}

Table 1. Baseline characteristics of the study subjects according to DR status. Data shown as mean \pm SD or $\%$, by Chi-squared test or general linear model (GLM). DR diabetic retinopathy; NPDR isolated non-proliferative diabetic retinopathy; $P D R$ proliferative diabetic retinopathy, $B M I$ body mass index; $S B P$ systolic blood pressure; $D B P$ diastolic blood pressure, $H b A 1 c$ glycated hemoglobin; $T C$ total cholesterol; $T G$ triglyceride; $H D L-C$ high density lipoprotein cholesterol; $L D L-C$ low density lipoprotein cholesterol; $B U N$ blood urea nitrogen; $\mathrm{CrCl}$ creatinine clearance; $A S T$ aspartate aminotransferase; $A L T$ alanine aminotransferase. 
(a) Glutamic acid (mg)

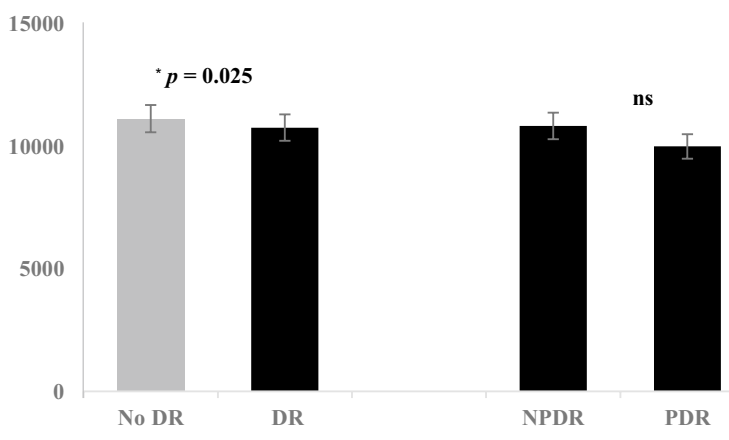

(c) Glutamic acid (\% total protein)
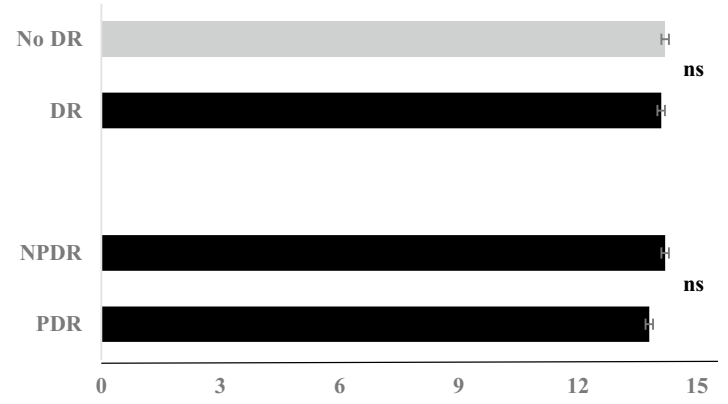

(b) Aspartic acid (mg)

9000

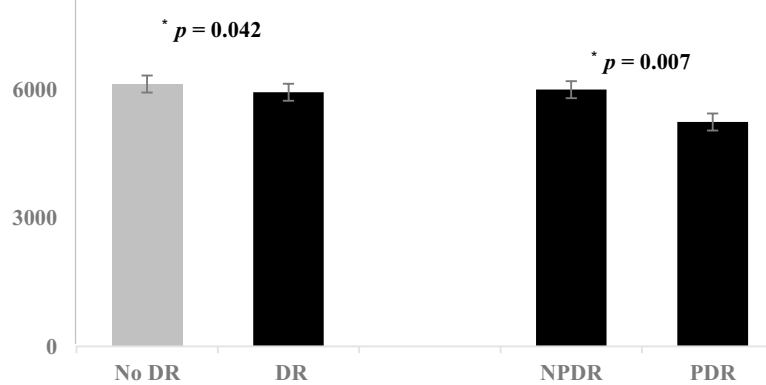

(d) Aspartic acid (\% total protein)

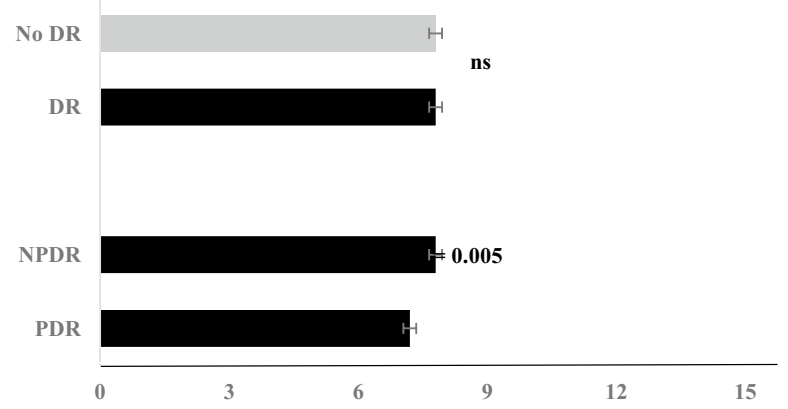

Figure 2. The differences of glutamic acid and aspartic acid intakes (mg or $\%$ total protein). The glutamic acid (a) and aspartic acid intakes (b) were lower in the DR group than those in the non-DR. There was no difference in proportion of total protein of glutamic acid (c) and aspartic acid (d). The absolute intake (b) and proportion of total protein (d) of aspartic acid were lower in the PDR group than those in the NPDR group. By general linear model (GLM), ns non-significant. NPDR non-proliferative diabetic retinopathy; PDR proliferative diabetic retinopathy; $D R$ diabetic retinopathy

\begin{tabular}{|c|c|c|c|c|}
\hline & \multirow[b]{2}{*}{ Total $(n=2067)$} & \multicolumn{3}{|l|}{ DR } \\
\hline & & Total DR $(n=731)$ & NPDR $(n=672)$ & PDR $(n=59)$ \\
\hline Median follow-up period, year & 5.0 & 5.3 & 5.4 & 5.3 \\
\hline Case/1000 Person-years $(\mathrm{PY})^{\star}$ & & 210.4 & 193.4 & 17.0 \\
\hline
\end{tabular}

Table 2. Incidence rate of DR. $D R$ diabetic retinopathy; NPDR isolated non-proliferative diabetic retinopathy; $P D R$ proliferative diabetic retinopathy. ${ }^{\star}$ PY: Person year, By Miettinen's (1974d) modification, as described in Epidemiologic Analysis with a Programmable Calculator, 1979. Results from OpenEpi, Version 3, open source calculator-PersonTime1.

were associated with DR. In this study, the baseline intakes of energy, glutamic acid, and aspartic acid were lower in the DR group than those in the non-DR group. However, when divided into tertiles according to glutamic acid and aspartic acid intakes, we observed no difference in the risk of DR incidence. Whereas, a low intake of aspartic acid was associated with a higher risk of developing PDR. While PDR incidence tended to increase with low glutamic acid intake, the difference was not statistically significant.

The pathogenesis of DR is mainly retinal vascular change. Chronic hyperglycemia induces basement membrane thickening, pericyte loss, and microvascular aneurysm or occlusion, resulting in a pathologic change of the blood-retinal barrier. The resultant retinal ischemia and increased vascular permeability cause vision-threatening $\mathrm{DR}^{17}$. The biochemical mechanisms involve the accumulation of sorbitol and advanced glycation end products (AGE), oxidative stress, protein kinase $\mathrm{C}$ activation, inflammation, and upregulation of the renin-angiotensin system (RAS) and vascular endothelial growth factor (VEGF) ${ }^{3}$. Recently, not only retinal vascular changes but also neuronal damage are thought to be important due to DR pathogenesis ${ }^{18}$. Although the mechanism of retinal neuron changes in DR is not yet understood, hypoxia-induced retinal neuron alteration might secondarily affect retinal tissue.

The role of dietary glutamic and aspartic acids in DR is not yet known. Although glutamic acid is the major excitatory neurotransmitter, excessive extracellular accumulation of glutamic acid damages neurons through excitotoxic mechanisms ${ }^{19}$. Glutamic neurotoxicity could induce the death of retinal ganglion cells (RGCs) in 


\begin{tabular}{|c|c|c|c|c|c|c|c|c|}
\hline & \multicolumn{3}{|c|}{ Tertiles of glutamic acid (mg) } & \multirow[b]{2}{*}{$p$ for trend } & \multicolumn{3}{|c|}{ Tertiles of aspartic acid $(\mathbf{m g})$} & \multirow[b]{2}{*}{$p$ for trend } \\
\hline & T1 & T2 & T3 & & T1 & T2 & T3 & \\
\hline \multicolumn{9}{|l|}{ DR } \\
\hline \multicolumn{9}{|c|}{ HR $(95 \%$ CI) } \\
\hline Crude & 1 [ref] & $1.00(0.78-1.28)$ & $0.90(0.66-1.22)$ & 0.436 & 1 [ref] & $1.04(0.81-1.34)$ & $0.90(0.66-1.23)$ & 0.411 \\
\hline Model 1 & 1 [ref] & $1.03(0.80-1.33)$ & $0.97(0.70-1.34)$ & 0.808 & 1 [ref] & $1.08(0.84-1.39)$ & $0.96(0.70-1.33)$ & 0.724 \\
\hline Model 2 & 1 [ref] & $1.02(0.79-1.24)$ & $1.01(0.82-1.24)$ & 0.617 & 1 [ref] & $1.07(0.83-1.39)$ & $0.93(0.67-1.29)$ & 0.551 \\
\hline Model 3 & 1 [ref] & $0.95(0.72-1.24)$ & $0.91(0.65-1.28)$ & 0.593 & 1 [ref] & $1.01(0.77-1.32)$ & $0.89(0.64-1.25)$ & 0.442 \\
\hline Model 4 & $1[\mathrm{ref}]$ & $0.97(0.73-1.28)$ & $0.94(0.66-1.33)$ & 0.711 & 1 [ref] & $1.01(0.77-1.33)$ & $0.88(0.62-1.25)$ & 0.427 \\
\hline \multicolumn{9}{|l|}{ NPDR } \\
\hline \multicolumn{9}{|c|}{ HR $(95 \%$ CI $)$} \\
\hline Crude & 1 [ref] & $1.00(0.77-1.29)$ & $0.94(0.69-1.29)$ & 0.681 & 1 [ref] & $1.04(0.80-1.35)$ & $0.97(0.71-1.34)$ & 0.794 \\
\hline Model 1 & 1 [ref] & $1.03(0.80-1.34)$ & $1.03(0.74-1.43)$ & 0.875 & 1 [ref] & $1.09(0.84-1.41)$ & $1.06(0.76-1.47)$ & 0.806 \\
\hline Model 2 & 1 [ref] & $1.02(0.83-1.30)$ & $0.99(0.71-1.39)$ & 0.942 & 1 [ref] & $1.08(0.82-1.41)$ & $1.03(0.74-1.43)$ & 0.956 \\
\hline Model 3 & 1 [ref] & $0.96(0.73-1.27)$ & $0.99(0.70-1.40)$ & 0.985 & 1 [ref] & $1.03(0.78-1.36)$ & $1.00(0.71-1.42)$ & 0.982 \\
\hline Model 4 & 1 [ref] & $0.97(0.73-1.30)$ & $1.01(0.71-1.45)$ & 0.901 & 1 [ref] & $1.01(0.76-1.35)$ & $0.98(0.68-1.39)$ & 0.859 \\
\hline \multicolumn{9}{|l|}{ PDR } \\
\hline \multicolumn{9}{|c|}{ HR (95\% CI) } \\
\hline Crude & 1 [ref] & $1.11(0.46-2.67)$ & $0.43(0.11-1.74)$ & 0.233 & 1 [ref] & $0.98(0.43-2.22)$ & $0.12(0.02-0.98)$ & 0.036 \\
\hline Model 1 & 1 [ref] & $1.07(0.44-2.61)$ & $0.41(0.10-1.67)$ & 0.188 & 1 [ref] & $0.94(0.41-2.15)$ & $0.11(0.01-0.91)$ & 0.028 \\
\hline Model 2 & 1 [ref] & $1.10(0.45-2.68)$ & $0.39(0.10-1.64)$ & 0.553 & 1 [ref] & $1.01(0.44-2.35)$ & $0.12(0.01-0.96)$ & 0.035 \\
\hline Model 3 & 1 [ref] & $0.90(0.36-2.27)$ & $0.28(0.06-1.25)$ & 0.083 & 1 [ref] & $0.83(0.35-2.01)$ & $0.09(0.01-0.79)$ & 0.015 \\
\hline Model 4 & $1[\mathrm{ref}]$ & $0.93(0.34-2.56)$ & $0.18(0.03-1.14)$ & 0.059 & 1 [ref] & $0.79(0.31-2.06)$ & $0.10(0.01-0.90)$ & \begin{tabular}{|l|}
0.024 \\
\end{tabular} \\
\hline
\end{tabular}

Table 3. Hazard ratios for diabetic retinopathy incidence according to tertiles of glutamic acid and aspartic acid intake (mg). By Cox proportional hazard models. DR, diabetic retinopathy; NPDR, isolated nonproliferative diabetic retinopathy; PDR, proliferative diabetic retinopathy. Model 1: adjusted for age and sex, Model 2: adjusted for Model 1 + HbA1C, duration of diabetes, Model 3: adjusted for Model $2+$ education, income, occupation, Model 4: adjusted for Model $3+\mathrm{CrCl}$, ALT, comorbidity.

DR or other ischemia-related ocular disease $\mathrm{s}^{20,21}$. Increased ROS production in the retina due to oxidative stress induced by hyperglycemia may impair glutamate-aspartate transporter (GLAST) function in Müller cells ${ }^{22}$. Müller cells regulate glutamate clearance in the retina through GLAST ${ }^{22}$. The level of asparagine and glutamine was increased in the aqueous humor of patients with DR, and highly activated alanine, aspartate, and glutamate metabolic pathway were identified in ${ }^{1} \mathrm{H}-\mathrm{NMR}$-based metabolomics analysis ${ }^{23}$.

Previous studies have shown the association of glutamic and aspartic acids with insulin resistance and secretion. The positive association between glutamic acid and waist circumference ${ }^{24,25}$ and visceral adipose tissue ${ }^{26}$ could be explained by the metabolism of branched-chain-amino-acids (BCAAs) in adipocytes to generate glutamic acid ${ }^{27}$. These findings suggest that glutamic acid was associated with increased insulin resistance, a finding consistent with reports in other studies that elevated plasma glutamic acid levels were associated with insulin resistance and abnormal fasting or 2-h glucose levels ${ }^{24,28}$. Moreover, a recent population-based large cohort study reported that nine amino acids, including glutamic acid and aspartic acid, were associated with decreased insulin secretion and elevated glucose level ${ }^{29}$. Increased insulin resistance and decreased insulin secretion can adversely affect blood glucose level, blood pressure, and blood lipid levels, increasing the risk of developing DR. However, the relationship between blood and retinal concentrations of glutamic and aspartic acids has not yet been reported.

In our study, the intakes of glutamic acid and aspartic acid did not affect the incidence of DR. This finding may be explained by the fact that the intake amount may not absolutely reflect blood levels. As non-essential amino acids, both glutamic acid and aspartic acid can be synthesized in the human body. Therefore, the blood levels of glutamic and aspartic acids are associated with both biosynthesis and dietary intake. Thus, the blood concentrations of glutamic and aspartic acids may be determined by biosynthesis in the body rather than intake.

Although glutamic acid and aspartic acid intakes did not affect DR development, aspartic acid intake affected DR severity, in which a lower intake was associated with PDR.

Alterations of some metabolites-related pathways in DR have been reported ${ }^{30}$. Because these alterations were related to the alanine-asparatic acid pathway and aspartic acid-asparagine pathway ${ }^{30}$, the influence of aspartic acid appears to be stronger.

This study has some limitations. First, dietary data on glutamic acid and aspartic acid intake were collected from dietary diaries. It is difficult to accurately extract and measure each dietary component in practice However, in this study, a trained dietitian used CAN Program for nutrient analysis. The CAN Program is widely using dietary assessment program in nutritional science in Korea. Second, the risk of developing DR was analyzed only from baseline intakes of glutamic and aspartic acids; however, whether baseline intake is representative of total intakes remains unknown. Therefore, dietary assessments were conducted every 3 years in this study, with 


\begin{tabular}{|c|c|c|c|c|c|c|c|c|}
\hline & \multicolumn{3}{|c|}{ Tertiles of glutamic acid (\% total protein) } & \multirow[b]{2}{*}{$p$ for trend } & \multicolumn{3}{|c|}{ Tertiles of aspartic acid (\% total protein) } & \multirow[b]{2}{*}{ P for trend } \\
\hline & T1 & T2 & T3 & & T1 & T2 & T3 & \\
\hline \multicolumn{9}{|l|}{ DR } \\
\hline \multicolumn{9}{|c|}{ HR $(95 \%$ CI $)$} \\
\hline Crude & 1 [ref] & $0.99(0.80-1.22)$ & $0.96(0.77-1.19)$ & 0.704 & 1 [ref] & $0.96(0.78-1.19)$ & $0.968(0.78-1.20)$ & 0.750 \\
\hline Model 1 & 1 [ref] & $1.00(0.81-1.23)$ & $0.96(0.77-1.19)$ & 0.733 & 1 [ref] & $0.95(0.77-1.18)$ & $0.970(0.78-1.20)$ & 0.758 \\
\hline Model 2 & 1 [ref] & $1.00(0.81-1.24)$ & $0.96(0.77-1.20)$ & 0.762 & 1 [ref] & $0.99(0.80-1.23)$ & $1.021(0.82-1.27)$ & 0.864 \\
\hline Model 3 & 1 [ref] & $0.98(0.79-1.23)$ & $0.89(0.71-1.12)$ & 0.362 & $1[\mathrm{ref}]$ & $0.99(0.79-1.25)$ & $0.995(0.79-1.25)$ & 0.962 \\
\hline Model 4 & $1[\mathrm{ref}]$ & $0.99(0.78-1.24)$ & $0.89(0.70-1.13)$ & 0.368 & 1 [ref] & $0.94(0.75-1.19)$ & $0.963(0.76-1.22)$ & 0.728 \\
\hline \multicolumn{9}{|l|}{ NPDR } \\
\hline \multicolumn{9}{|c|}{ HR $(95 \%$ CI $)$} \\
\hline Crude & 1 [ref] & $1.03(0.82-1.28)$ & $0.99(0.79-1.23)$ & 0.922 & 1 [ref] & $1.04(0.83-1.30)$ & $1.03(0.83-1.29)$ & 0.757 \\
\hline Model 1 & 1 [ref] & $1.03(0.83-1.29)$ & $0.99(0.79-1.24)$ & 0.964 & 1 [ref] & $1.03(0.83-1.29)$ & $1.04(0.83-1.30)$ & 0.731 \\
\hline Model 2 & 1 [ref] & $1.04(0.83-1.30)$ & $1.00(0.80-1.25)$ & 0.971 & 1 [ref] & $1.08(0.86-1.35)$ & $1.10(0.88-1.37)$ & 0.410 \\
\hline Model 3 & 1 [ref] & $1.03(0.82-1.29)$ & $0.93(0.73-1.19)$ & 0.624 & 1 [ref] & $1.08(0.85-1.37)$ & $1.08(0.85-1.36)$ & 0.526 \\
\hline Model 4 & 1 [ref] & $1.04(0.82-1.32)$ & $0.95(0.74-1.21)$ & 0.711 & & $1.04(0.82-1.32)$ & $1.05(0.82-1.33)$ & 0.711 \\
\hline \multicolumn{9}{|l|}{ PDR } \\
\hline \multicolumn{9}{|c|}{ HR (95\% CI) } \\
\hline Crude & 1 [ref] & $0.68(0.31-1.48)$ & $0.77(0.36-1.64)$ & 0.434 & 1 [ref] & $0.43(0.19-0.94)$ & $0.43(0.20-0.94)$ & 0.017 \\
\hline Model 1 & 1 [ref] & $0.68(0.31-1.47)$ & $0.76(0.35-1.62)$ & 0.411 & 1 [ref] & $0.42(0.19-0.92)$ & $0.42(0.19-0.93)$ & 0.015 \\
\hline Model 2 & 1 [ref] & $0.72(0.33-1.57)$ & $0.75(0.35-1.61)$ & 0.416 & 1 [ref] & $0.46(0.21-1.02)$ & $0.46(0.21-1.02)$ & 0.031 \\
\hline Model 3 & 1 [ref] & $0.63(0.27-1.47)$ & $0.67(0.29-1.51)$ & 0.283 & 1 [ref] & $0.38(0.16-0.92)$ & $0.44(0.19-1.03)$ & 0.027 \\
\hline Model 4 & 1 [ref] & $0.55(0.22-1.34)$ & $0.47(0.19-1.20)$ & 0.091 & 1 [ref] & $0.26(0.09-0.70)$ & $0.39(0.16-0.96)$ & 0.013 \\
\hline
\end{tabular}

Table 4. Hazard ratio for diabetic retinopathy incidence according to tertiles of glutamic acid and aspartic acid intake (\% total protein). By Cox proportional hazard models. DR diabetic retinopathy; NPDR isolated non-proliferative diabetic retinopathy; $P D R$ proliferative diabetic retinopathy. ${ }^{\star}$ Model 1 , adjusted for age and sex. ${ }^{* \star}$ Model 2, adjusted for Model $1+\mathrm{HbA1C}$, duration of diabetes mellitus. ${ }^{\star * \star}$ Model 3, adjusted for Model $2+$ education, income, occupation. ${ }^{\star \star \star \star}$ Model 4 , adjusted for Model $3+\mathrm{CrCl}$, ALT, comorbidity.

no difference in the intakes of glutamic and aspartic acids compared to those at baseline. Recent studies have assessed the relationship between DR and metabolites. To our knowledge, the present study is the first to analyze the association of DR and metabolites absorbed by dietary intake.

In conclusion, the dietary glutamic acid and aspartic acid did not affect the incidence of DR. However, aspartic acid intake was negatively correlated with PDR. The metabolites absorbed by dietary intake may not directly affect the concentrations in blood and body fluids.

\section{Methods}

The data for this study were derived from the Korea National Diabetes Program (KNDP) cohort study performed at 12 hospitals. The KNDP is a prospective, multicenter, observational study evaluating Korean patients with T2DM (NCT 01212198). The first patients were enrolled in May 2006 and the follow-up period was defined as the time between baseline and March 2014. Detailed descriptions of the KNDP cohort study design and data collection have been described elsewhere ${ }^{10}$.

Among 4601 T2DM patients, we excluded those with missing values in their dietary history (1266 energy values and 12 amino acid values) and NPDR and PDR data $(n=650)$. Participants with total energy intake outside the limits $(<500$ and $>5000 \mathrm{kcal} /$ day $)(\mathrm{n}=1)$ and with amino acid levels that were unlikely to be accurate $(n=14)$ were also excluded. Participants who had been diagnosed with DR before the study period began were also excluded $(n=229)$. After applying these exclusion criteria, this study included 2429 subjects ( 1189 men, 878 women), of whom 2067 completed the study (Fig. 1).

This was a multicenter, prospective cohort study. The primary outcome was the incidence of DR during the follow-up period. The baseline data were collected based on sociodemographic (age, sex, education level, income, occupation, and smoking and alcohol consumption), clinical characteristics (body mass index, blood pressure), biochemical examination (glycemic control, lipid control, renal function, and liver function), DM duration, medical history (hypertension [HTN], dyslipidemia, cardiovascular or cerebrovascular disease), and dietary intakes. Dietary assessment was performed by a trained dietitian using a 3-day food records. The baseline energy, glutamic acid, and aspartic acid intakes were assessed and the absolute intake (mg) and proportion of total protein (\%) determined. Nutrient analysis was performed using Computer-Aided Nutritional (CAN) analysis version 3.0 (Korean Nutrition Society, Seoul, South Korea). Dietary assessment was conducted every 3 years. DR was assessed by color fundus photography (FF 540 Plus; Carl Zeiss Meditech, Jena, Germany) and optical coherence tomography (HD-OCT; Carl Zeiss Meditech, Dublin, CA, USA) at baseline. All subjects underwent DR assessment annually. In accordance with Early Treatment Diabetic Retinopathy Study (ETDRS) criteria, DR was graded into three categories: non-DR, non-proliferative diabetic retinopathy (NPDR), or PDR ${ }^{11,12}$. Two 
or more ophthalmologists classified the DR status based on the examination results. Discordance between the evaluators was resolved by a review of the images to agree on the final interpretation.

The effects of glutamic acid and aspartic acid intake on DR incidence were analyzed by dividing the intake amount (mg or \% protein) into tertiles. The HR for DR incidence in the middle and highest tertiles were compared to the lowest tertile.

The baseline characteristics of the subjects in the three groups (no DR, NPDR, PDR) are expressed as means \pm standard deviations (SD) for continuous variables and as percentages for categorical variables. To compare the characteristics among groups, general linear models (GLMs) were used for continuous variables and chi-squared tests for categorical variables. The HRs and 95\% confidence interval (CIs) for incident NPDR, PDR, and DR were calculated according to tertiles of glutamic and aspartic acid intakes ( $\mathrm{mg}$ or \% protein) by Cox proportional hazard models. In the multivariate-adjusted models, Model 1 was adjusted for age and sex, while Model 2 was adjusted for the covariates in Model 1 plus glycated hemoglobin (HbA1c) level, and DM duration. Model 3 was adjusted for the covariates included in Model 2 plus education, income, and occupation. Model 4 was additionally adjusted for creatinine clearance $(\mathrm{CrCl})$, alanine aminotransferase (ALT) level, and comorbidities. Statistical significance was defined as $p<0.05$. SAS (version 9.4; SAS Institute Inc., Cary, NC, USA) was used for all statistical analyses.

The institutional review boards of all investigational centers granted ethical approval for the study and informed consent was obtained from all subjects (Kyung Hee Medical Center IRB 1415-04). All methods were performed in accordance with relevant guidelines and regulations.

\section{Data availability}

The datasets generated during and/or analyzed during the current study are available from the corresponding author on reasonable request.

Received: 8 May 2020; Accepted: 20 January 2021

Published online: 31 March 2021

\section{References}

1. Ding, J. \& Wong, T. Y. Current epidemiology of diabetic retinopathy and diabetic macular edema. Curr. Diab. Rep. 12, 346-354 (2012).

2. Song, S. J. \& Wong, T. Y. Current concepts in diabetic retinopathy. Diabetes Metab. J. 38, 416-425 (2014).

3. Cheung, N., Mitchell, P. \& Wong, T. Y. Diabetic retinopathy. Lancet 376, 124-136 (2010).

4. Resnikoff, S. et al. Global data on visual impairment in the year 2002. Bull. World Health Organ 82, 844-851 (2004).

5. Congdon, N. G., Friedman, D. S. \& Lietman, T. Important causes of visual impairment in the world today. JAMA 290, 2057-2060 (2003).

6. Rhee, S. Y. et al. Diabcare Asia 2001-Korea country report on outcome data and analysis. Korean J. Intern. Med. 20, 48-54 (2005).

7. Byun, S. H. et al. Screening for diabetic retinopathy and nephropathy in patients with diabetes: A nationwide survey in Korea. PLoS ONE 8, e62991 (2013).

8. Jenkins, A. J. et al. Biomarkers in diabetic retinopathy. Rev. Diabet. Stud. 12, 159-195 (2015).

9. Rhee, S. Y. et al. Plasma glutamine and glutamic acid are potential biomarkers for predicting diabetic retinopathy. Metabolomics 14, 89 (2018).

10. Rhee, S. Y. et al. Prevalence of chronic complications in korean patients with type 2 diabetes mellitus based on the korean national diabetes program. Diabetes Metab. J. 35, 504-512 (2011).

11. Wilkinson, C. P. et al. Proposed international clinical diabetic retinopathy and diabetic macular edema disease severity scales. Ophthalmology 110, 1677-1682 (2003).

12. Wu, L. et al. Classification of diabetic retinopathy and diabetic macular edema. World J. Diabetes 4, 290-294 (2013).

13. Yau, J. W. et al. Global prevalence and major risk factors of diabetic retinopathy. Diabetes Care 35, 556-564 (2012).

14. Cheung, N. et al. Diabetic retinopathy and the risk of coronary heart disease: The Atherosclerosis Risk in Communities Study. Diabetes Care 30, 1742-1746 (2007).

15. Gerstein, H. C. et al. Diabetic retinopathy, its progression, and incident cardiovascular events in the ACCORD trial. Diabetes Care 36, 1266-1271 (2013).

16. Cheung, N. et al. Is diabetic retinopathy an independent risk factor for ischemic stroke?. Stroke 38, 398-401 (2007).

17. Ahsan, H. Diabetic retinopathy-biomolecules and multiple pathophysiology. Diabetes Metab. Syndr. 9, 51-54 (2015).

18. Puro, D. G. Diabetes-induced dysfunction of retinal Muller cells. Trans. Am. Ophthalmol. Soc. 100, 339-352 (2002).

19. Bai, N. et al. NMDA receptor subunits have different roles in NMDA-induced neurotoxicity in the retina. Mol. Brain 6, 34 (2013).

20. Hernandez, C. \& Simo, R. Neuroprotection in diabetic retinopathy. Curr. Diab. Rep. 12, 329-337 (2012).

21. Kaur, C., Foulds, W. S. \& Ling, E. A. Hypoxia-ischemia and retinal ganglion cell damage. Clin. Ophthalmol. 2, 879-889 (2008).

22. Ma, M. et al. High glucose-induced TRPC6 channel activation decreases glutamate uptake in rat retinal muller cells. Front. Pharmacol. 10, 1668 (2019).

23. Jin, H. et al. Metabolic characterization of diabetic retinopathy: An (1)H-NMR-based metabolomic approach using human aqueous humor. J. Pharm. Biomed. Anal. 174, 414-421 (2019).

24. Cheng, S. et al. Metabolite profiling identifies pathways associated with metabolic risk in humans. Circulation 125, 2222-2231 (2012).

25. Maltais-Payette, I. et al. Circulating glutamate level as a potential biomarker for abdominal obesity and metabolic risk. Nutr. Metab. Cardiovasc. Dis. 29, 1353-1360 (2019).

26. Boulet, M. M. et al. Alterations of plasma metabolite profiles related to adipose tissue distribution and cardiometabolic risk. Am. J. Physiol. Endocrinol. Metab. 309, E736-E746 (2015).

27. Maltais-Payette, I. et al. Circulating glutamate concentration as a biomarker of visceral obesity and associated metabolic alterations. Nutr. Metab. (Lond) 15, 78 (2018).

28. Seibert, R. et al. Relationship between insulin resistance and amino acids in women and men. Physiol. Rep. 3, e12392 (2015).

29. Vangipurapu, J. et al. Nine amino acids are associated with decreased insulin secretion and elevated glucose levels in a 7.4-year follow-up study of 5181 finnish men. Diabetes 68, 1353-1358 (2019).

30. Sumarriva, K. et al. Arginine and carnitine metabolites are altered in diabetic retinopathy. Invest. Ophthalmol. Vis. Sci. 60, 31193126 (2019). 


\section{Acknowledgements}

This study was supported by a grant from the Korea Healthcare Technology R\&D Project, Ministry of Health and Welfare Republic of Korea (HI10C2020).

\section{Author contributions}

S.Y.R. contributed to the conception and design of the study, researched data, and reviewed/edited the manuscript. S.Y.P., J.E.K., J.I.S. and D.Y.K. helped with the research of the data, and its interpretation. S.Y.P. drafted the manuscript. J.E.K. and D.Y.K. performed the statistical analyses and data interpretation. H.J.L., S.C., and J.T.W. helped to design the study and contributed via discussions. All authors read and approved the final manuscript.

\section{Competing interests}

The authors declare no competing interests.

\section{Additional information}

Correspondence and requests for materials should be addressed to S.Y.R. or H.L.

Reprints and permissions information is available at www.nature.com/reprints.

Publisher's note Springer Nature remains neutral with regard to jurisdictional claims in published maps and institutional affiliations.

(c) (i) Open Access This article is licensed under a Creative Commons Attribution 4.0 International License, which permits use, sharing, adaptation, distribution and reproduction in any medium or format, as long as you give appropriate credit to the original author(s) and the source, provide a link to the Creative Commons licence, and indicate if changes were made. The images or other third party material in this article are included in the article's Creative Commons licence, unless indicated otherwise in a credit line to the material. If material is not included in the article's Creative Commons licence and your intended use is not permitted by statutory regulation or exceeds the permitted use, you will need to obtain permission directly from the copyright holder. To view a copy of this licence, visit http://creativecommons.org/licenses/by/4.0/.

(C) The Author(s) 2021 